\title{
V-Series Nerve Agent VX
}

National Cancer Institute

\section{Source}

National Cancer Institute. V-Series Nerve Agent VX. NCI Thesaurus. Code C161531.

An org anic thiophosphate nerve agent that is a persistent, odorless, amber-colored oily liquid at room temperature that is absorbed into the body through inhalation or skin contact, and is an acetylcholinesterase inhibitor and neurotoxin. 